\title{
Mitteilungen der VMTB
}

VSRN 2016

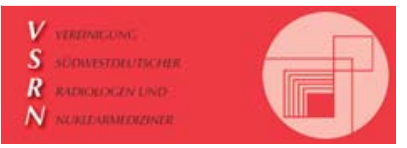

Die Vereinigung der Südwestdeutschen Radiologen und Nuklearmediziner tagten zum 26. Mal im Kongresszentrum in Karlsruhe. Das Programm für MTRA war gefüllt mit vielen Themen zur Schnittbildtechnik im MRT und der Projektionsradiografie.

Die Schnittbildtechnik konzentrierte sich, neben den Grundlagen der MRT-Technik und Artefakten, auf die Untersuchungsmethoden der unteren Extremität, des Abdomens und der Wirbelsäule. Die Sitzungen der Projektionsradiografie beschäftigten sich mit der konventionellen Röntgendiagnostik des Körperstammes und Metallimplantaten, die im Bereich der Wirbelsäule eingebracht werden können.

Begleitet wurde das Fort- und Weiterbildungsprogramm von einer umfangreichen Industrieausstellung, die einen intensiven Austausch zwischen Vertretern der Industrie und den Teilnehmerinnen und Teilnehmern zu innovativen Weiterentwicklungen in der Medizin ermöglichte.

Mit einer Teilnehmerzahl von knapp 1300 Personen, davon knapp 100 MTRA stellt die Veranstaltung die größte fachübergreifende radiologische Regionalveranstaltung in Deutschland dar.

Die kommende Veranstaltung in 2017 wird Sie, wie auch der Deutsche Röntgenkongress, mit neuen Wegen überraschen. Ich darf Sie alle jetzt schon einladen sich den Termin für das Jahr 2017 vorzumerken. Die nächste Fort- und Weiterbildungsveranstaltung der VSRN e.V. findet am 2. und 3. Februar 2017 im Kongresszentrum Baden-Baden statt. Weitere Informationen und finden Sie auch unter www.vsrn.de.

Claus Becker

Vorstandsvorsitzender VMTB

\section{Leipziger Allerlei: spannende und wesentliche Themen für MTRA}

Volles Haus beim 6. Leipziger Allerlei - Fortbildungsveranstaltung für MTRA am Universitätsklinikum Leipzig am 30.Januar 2016.

Das „Leipziger Allerlei“ der VMTB ist eine Erfolgsgeschichte: Bereits im 6.Jahr ist es gelungen, den Hörsaal der Universitätsklinik in Leipzig zu füllen. Damit ist es eine der größten MTRA-Veranstaltungen überhaupt in ganz Deutschland.
Über 320 MTRA und MTRA-Schüler bevölkerten den Hörsaal und das angrenzende lichtdurchflutete Foyer und alle waren rundum begeistert von den Themen: „Es ist schön, dass auf einer Fortbildungsveranstaltung auch mal grundlegende Themen besprochen werden“, so eine Teilnehmerin. „Und alle Referenten haben sie spannend rübergebracht.“

Ein Referententeam aus Leipzig eröffnete das Programm mit dem Thema der „Bildbetrachtung und Einstelltechnik“ aus dreierlei Perspektiven: der Unfallchirurgie, der Orthopädie und der Kinderradiologie. Hier konnten also einige Teilnehmer auch mal in radiologische Nachbargebiete reinschnuppern.

Nach einer Kaffeepause ging es dann um die „Computertomografie“. Herr Alex Riemer, MTRA aus Mönchengladbach, diskutierte hierbei mit dem Auditorium, ob es denn immer höher, schneller und weiter sein muss oder worauf es denn nun wirklich ankommt.

Gestärkt aus der Mittagspause zurückgekehrt, ging es um das „Abdomen“. Kollegen aus Regensburg, München und Jena erläuterten die Diagnostik des Magen-Darm-Traktes, des Urogenital-Systems und der Leber, Milz und Aorta.

Schließlich endete der Fortbildungstag mit einem „Update MRT“. Von MRT-Sicherheit über Neuigkeiten im MRT bis zu Artefakten kamen die Teilnehmer auch hier voll auf ihre Kosten.

Einen herzlichen Dank an dieser Stelle geht an alle Firmen, die das 6. Leipziger Allerlei tatkräftig unterstützt haben. Auch allen Referenten danken wir sehr herzlich für ihr Engagement.

Am 27. Januar 2017 findet das 7. Leipziger Allerlei wieder im Universitätsklinikum Leipzig statt. Themen werden dann „Kontrastmittel“, „Sportmedizin“, „das Becken“, und „Neuigkeiten von...“ sein.

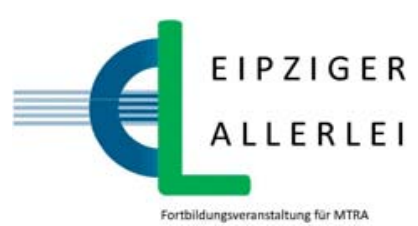

(c) DRG.

Wir freuen uns schon jetzt Sie dort wieder begrüßen zu dürfen. Infos und Anmeldung finden Sie ab Herbst 2016 auf unserer Homepage www.vmtb.de.

Nina Keil

Pressestelle der DRG
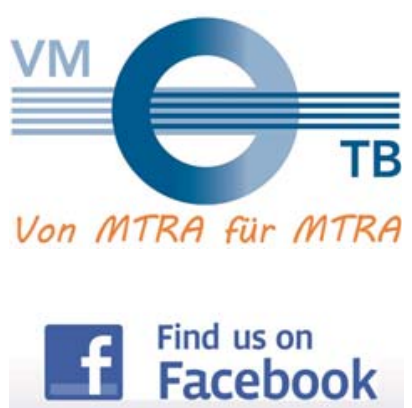
Werden Sie Fan vom VMTB unter
www.facebook.de/vmtb.de

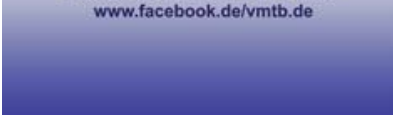

Geschäftsstelle:

VMTB - Vereinigung der Medizinisch-Technischen Berufe in der DRG

Frau Sonja Müller

Tel.: + 49 (0) 3091607015

Fax: +49 (0) 3091607022

E-Mail: mueller@drg.de

Frau Nina Keil

Tel.: +49 (0) 3091607025

Fax: +49 (0) 3091607022

E-Mail: keil@drg.de

Ernst-Reuter-Platz 10

10587 Berlin

www.vmtb.de

Facebook:

www.facebook.de/vmtb.de 
Einladung zum 7. Rheinisch-westfälischen MTRA-Symposium

Rheinisch-Westfälisches (C) DRG.

Sehr geehrte Damen und Herren,

als Vertreter der Rheinisch-Westfälischen Röntgengesellschaft (RWRG) und der Vereinigung der medizinisch-technischen Berufe in der Deutschen Röntgengesellschaft (VMTB) möchten wir Sie herzlich zum 7.Rheinisch-westfälischen MTRASymposium einladen und freuen uns, Sie am 1.7. 2016 im Factory-Hotel in Münster willkommen heißen zu dürfen.

Systematisch und „up to date“ werden wir uns in diesem Jahr mit der Bildgebung rund um den Bereich der Wirbelsäule sowie des Abdomens beschäftigen. Gemeinsam werden klinische Partner, MTRA und Radiologen anatomische Grundlagen, relevante Krankheitsbilder, Bildbefunde sowie Untersuchungs- und Interventionstechniken vorstellen.

Neben praxisrelevanten Aspekten möchten wir Ihnen darüber hinaus Ein- und Ausblicke geben in die neuesten Entwicklungen der Computerund Magnetresonanztomografie sowie des digitalen Röntgens.

Im letzten Teil der Veranstaltung widmen wir uns dem direkten Arbeitsumfeld. Hier werden Sie die Möglichkeit haben, den kommunikativen Umgang mit Ihren Patienten zu trainieren. Ein weiterer Beitrag soll Ihnen mehr Klar- und Rechtsicherheit rund um das Aufklärungsgespräch geben.

Mitdenken und hinschauen heißt es abschließend, wenn Sie eingeladen sind, klinische Fälle im Quiz zu lösen und zu diskutieren.

Wir freuen uns, Sie in Münster begrüßen zu dürfen.

Prof. Dr. med. J. Wessling, Chefarzt der Klinik für Radiologie, Clemenshospital, Münster Prof. Dr. med. C. Bremer, Chefarzt der Klinik für Radiologie, St.-Franziskus-Hospital, Münster Annette Saur, Vorstand VMTB, Essen

\section{Einladung nach Kempten}

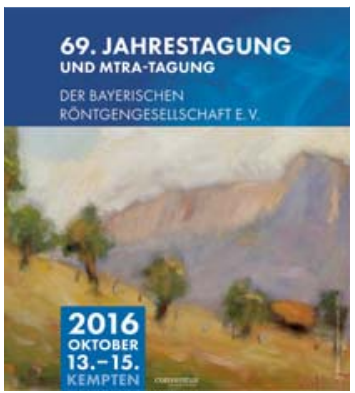

(c) DRG.
Liebe Kolleginnen und Kollegen,

wir möchten Sie herzlich zur 69. Jahrestagung und MTRA-Tagung der bayerischen Röntgengesellschaft vom 13.-15.Oktober nach Kempten einladen.

Um sowohl der medizinischen als auch der technischen Entwicklung Rechnung zu tragen ist Weiterbildung unerlässlich. Unsere Jahrestagung bietet Fortbildung aus einem breiten Querschnitt vieler aktueller Themen. Es erwarten Sie Sitzungen aus den Themenblöcken Abdomen, Herz, Muskuloskelletalem System, Neuroradiologie, Kinderradiologie, Intervention und Hybridbildgebung. Alles immer in Hinblick auf die praktische
Nützlichkeit im radiologischen Alltag in Klinik und Praxis.

Dazu wird es auch in diesem Jahr wieder ein spezielles Programm für MTRA sowie die bewährten „Fit für den Facharzt“-Kurse geben, letztere mit dem Schwerpunkt auf Onkologie.

Ergänzt wird das breitgefächerte Programm mit Abrechnungsseminaren (auch für entsprechend tätige MTRA und Mitarbeiter aus den Sekretariaten etc., speziell auch zu Intervention) und einem Aktualisierungskurs für die Fachkunde nach Röntgenverordnung sowie durch eine begleitende Industrieausstellung.

Die Tagung findet in modernen Veranstaltungsräumen der BigBox im Zentrum von Kempten statt und bietet damit ein professionelles Umfeld für Ihre Fortbildung. Aktuelle Informationen finden sie unter www.brg-kongress.de.

Wir freuen uns auf Ihren Besuch und darauf, Sie im Oktober in Kempten begrüßen zu können!

Mit herzlichen Grüßen

Prof. Dr. med. Andrik Aschoff

Tagungsleitung 2016

Prof. Dr. med. Michael Uder

1. Vorsitzender der BRG

Einladung zum 9. RadiologieKongressRuhr, 3. - 5.11.2016 in Bochum

Sehr geehrte Damen und Herren,

nachdem im Frühjahr der 97. Deutsche Röntgenkongress in Leipzig als bundesweit größter Fortbildungskongress stattfindet, freuen wir uns Ihnen im Herbst mit dem 9. RadiologieKongressRuhr in Bochum den bundesweit zweitgrößten Fortbildungskongress mit interessanten Themen, Referenten und neuesten Entwicklungen anbieten zu können.

Da der Innovationsgehalt innerhalb der Kongressthemen stark variiert, haben wir in Vorbereitung des Kongresses 2016 zunächst wieder geschaut, in welchen Disziplinen sich am meisten tut bzw. welche Themen beim RKR (oder auch auf anderen Kongressen) zuletzt tendenziell zu kurz gekommen sind. Bezogen auf das von der RheinischWestfälischen Röntgengesellschaft und der Deut-

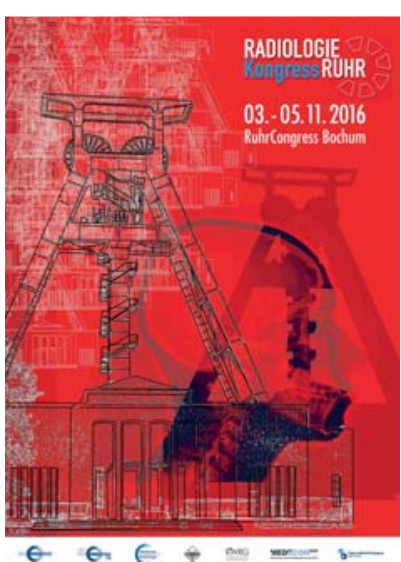

(C) DRG. 
schen Röntgengesellschaft gemeinsam verantwortete Hauptprogramm wurden für den RKR 2016 demnach folgende Schwerpunkte gesetzt: Uroradiologie, Interventionen, Onkologie, Abdominelle Bildgebung (Tipps \& Tricks), Neuroradiologie (inkl. Neuro-Cases im Dialog), Muskuloskelettale Radiologie, Thoraxdiagnostik, Kinderradiologie.

Unter der Federführung der VMTB wird es zudem natürlich auch wieder einen eigenen MTRA-Fortbildungstag sowie einen MTRA-Schülertag geben. Technische Innovationen bilden das Leitmotiv der Industriesymposien und Industrieworkshops. Hier besteht für Unternehmen die Möglichkeit, eigene Programminhalte zu platzieren.

Neben der reinen Fortbildung präsentiert sich der RadiologieKongressRuhr ansonsten auch in seiner 9.Auflage als überregionale Kommunikationsplattform. Ausführliche Kongresspausen sowie der traditionelle Empfang „Medizin trifft Industrie“ der Stadt Bochum am ersten Veranstaltungstag bieten viel Raum und Zeit zur allgemeinen Kontaktpflege.

In diesem Sinne würden wir uns freuen, Sie auf dem RKR 2016 begrüßen zu dürfen und verbleiben

mit den besten Grüßen

Prof. Dr. Dieter Liermann

Prof. Dr. Werner Weber

Prof. Dr. Johannes Wessling

\section{Schon Urlaub gebucht?}

Warum nicht mal das Nützliche mit dem Angenehmen verbinden? - Gelegenheit dazu bietet auch in diesem Jahr wieder das „Greifswalder Ryck-Symposium“.

Den nützlichen Teil bildet am 2. und 3. September wieder ein breit gefächertes Programm für MTRA mit spannenden und interessanten Vorträgen und dieses Mal auch ein Hands-On-Workshop. Das Programm finden Sie unter www.vmtb.de/ Veranstaltungen.

Zum angenehmen Teil zählt auf jeden Fall wieder ein Segeltörn, der inzwischen beim Greifswalder Ryck-Symposium schon zur Tradition gehört. Die „Weiße Düne“ ist bereit für die Teilnehmer. Wem das aber nicht genug Vergnügen ist, dem seien ein paar Tage Urlaub in Greifswald und Umgebung empfohlen.

Viele weitere Informationen und Anregungen bieten die Internetseiten der Touristikbüros.

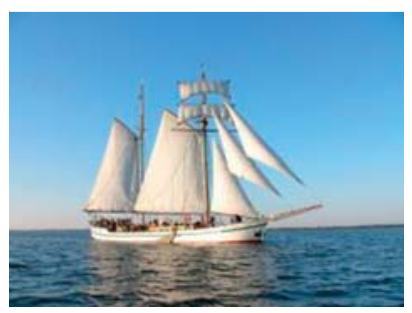

Segeltörn beim Greifswalder Ryck-Symposium. (c) Weisseduene.com.
Also, ein Vermerk im Terminkalender: 2./3.September „Ryck-Symposium“ in Greifswald lohnt sich auf jeden Fall.

Brigitte Olbrich

VMTB kooptiertes Vorstandsmitglied

\section{Radiologiekongress Nord - Eine gelungene}

\section{Premiere}

Am 5. und 6. Februar 2016 fand erstmalig der Radiologiekongress Nord in Hamburg statt.

Der Radiologiekongress Nord ging unter Leitung des Kongresspräsidenten Professor Fischbach aus der gemeinsamen Jahrestagung der Norddeutschen Röntgengesellschaft und der Röntgengesellschaft von Niedersachsen, Bremen und Sachsen-Anhalt hervor.

In dieser Neuauflage wurde nun auch für Norddeutschland die Möglichkeit einer eigenen Fortbildungsveranstaltung der VMTB für MTRA geschaffen. Sie stand unter dem Motto: „Aktuelle Herausforderungen“.

Herr Apel, Verantwortlicher für das MTRA-Programm und Vorstandsmitglied der VMTB, begrüßte die Teilnehmer aus den unterschiedlichsten Bundesländern, von Schleswig-Holstein bis Bayern, im Vortragssaal der Handwerkskammer Hamburg und eröffnete die Veranstaltung.

12 Referenten sorgten mit ihren Vorträgen für ein abwechslungsreiches, interessantes und kurzweiliges Programm. Die Themen kamen aus fast allen Bereichen der Radiologie, wie z.B. digitale Radiografie, CT, MRT, sowie Ausblicke in die Gegenwart und Zukunft, sodass für jeden etwas dabei war.

Die beiden letzten Vorträge am Samstag wurden von den Teilnehmenden mit besonderer Spannung erwartet. Der Referent sprach über den Fachkräftemangel im MTRA-Beruf und löste damit eine rege Diskussion aus. Dabei wurde aber auch deutlich, dass das Thema eine relevante Bedeutung in der Auseinandersetzung mit dem MTRA-Beruf hat. Im letzten Vortrag der Veranstaltung versuchte Professor Jung den Teilnehmern die Wichtigkeit des MTRA-Berufes zu verdeutlichen.

MTRA am Ultraschall war die Empfehlung.

In der 3.Etage präsentierten an beiden Fortbildungstagen 26 Firmen ihre Produkte auf der Industrieausstellung und standen den Teilnehmern Rede und Antwort.

An dieser Stelle sei allen Referenten und Firmenvertretern nochmals herzlich gedankt.

Sie haben einen entscheidenden Beitrag zum Erfolg der Veranstaltung beigetragen. Ein herzliches Dankeschön gilt auch den Teilnehmern, die ein interessiertes und aufmerksames Publikum waren.

Marcel Apel

VMTB Vorstand

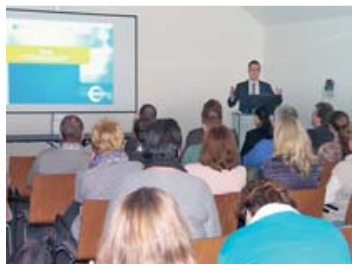

Voller Saal beim NDRG in Hamburg, Begrüßung Marcel Apel. (c) Müller. 